\title{
Function and organization: Comparing the mechanisms of protein
} synthesis and natural selection

Phyllis McKay Illari and Jon Williamson

The corresponding author is Phyllis Illari

\author{
Philosophy Department \\ University of Kent \\ Canterbury \\ Kent \\ CT2 7NZ \\ p.mckay@kent.ac.uk \\ j.williamson@kent.ac.uk
}




\title{
Function and organization: Comparing the mechanisms of protein synthesis and natural selection
}

\begin{abstract}
In this paper, we compare the mechanisms of protein synthesis and natural selection. We identify three core elements of mechanistic explanation: functional individuation, hierarchical nestedness or decomposition, and organization. These are now well understood elements of mechanistic explanation in fields such as protein synthesis, and widely accepted in the mechanisms literature. But Skipper and Millstein have argued (2005) that natural selection is neither decomposable nor organized. This would mean that much of the current mechanisms literature does not apply to the mechanism of natural selection.

We take each element of mechanistic explanation in turn. Having appreciated the importance of functional individuation, we show how decomposition and organization should be better understood in these terms. We thereby show that mechanistic explanation by protein synthesis and natural selection are more closely analogous than they appear - both possess all three of these core elements of a mechanism widely recognized in the mechanisms literature.
\end{abstract}

Keywords: mechanism, function, decomposition, organization, natural selection, protein synthesis.

\section{Introduction: the problem}

There has been great progress in understanding mechanistic explanations in particular domains, but this progress needs to be extended to cover all sciences. We will advance that project here by comparing protein synthesis and natural selection. Both are called mechanisms, but the fields differ significantly, and Skipper and Millstein have recently argued that natural selection is not captured by the accounts of mechanisms currently available. They make the challenge: 'We think the basic resources for characterizing the mechanism of natural selection may be found in the new mechanistic philosophy. And we urge the proponents of the philosophy to explore the directions for further work we suggest.' (Skipper and Millstein 2005 p345.) Barros (2008) has responded to their challenge that natural selection is not regular enough to satisfy current accounts. ${ }^{1}$ Here, we take up a different aspect of their challenge, responding to their arguments that natural selection does not decompose into organized parts.

We take mechanisms to have three core elements: functional individuation, decomposition and organization. Mechanistic explanation begins with a description of the phenomenon to be explained. The phenomenon is explained by being decomposed into lower-level components. Perhaps the functioning of lower-level components is in turn explained by further decomposition into yet lower-level components. So mechanisms come to be hierarchically nested. ${ }^{2}$ Organization is the final element of this brief picture. The phenomenon is not explained until it is shown how the components are

\footnotetext{
${ }^{1}$ Machamer also now agrees that the regularity requirement in Machamer, Darden and Craver (MDC 2000) is too strong. (2004 p37 Footnote 1.)

${ }^{2}$ In this hierarchy, higher levels are often important. They can be crucial in identifying and describing the phenomenon itself, for example. We do not mean to imply that only the lower levels are involved in the explanation of a phenomenon. The explanation might consist of fitting the phenomenon into an entire nested hierarchy.
} 
organized in order to produce the phenomenon of interest. The actual process of mechanism discovery is far messier than this, of course, but these three elements are involved in the mechanisms discovered.

These three elements are vital to a view of mechanistic explanation now quite uncontroversial in the literature that examines such mechanisms as protein synthesis. (See MDC 2000; Bechtel and Abrahamsen 2005, 2008; Craver 2007; Darden 2006.) But Skipper and Millstein's arguments are not negligible: it is indeed hard to see how explanations offered by natural selection decompose into organized parts. Skipper and Millstein accept the functional individuation of natural selection, but we also examine this because it is important to understanding both decomposition and organization. So we take all three elements in turn in S2, S3 and S4, and argue that natural selection, like protein synthesis, possesses all three elements. In the process, we develop an understanding of both decomposition and organization. Once these three elements are better understood, it becomes clear that these elements of mechanistic explanation in the current mechanisms literature do apply to the mechanism of natural selection.

In this paper, we will move between claims about mechanistic explanation, and claims about the mechanisms themselves. This might be thought problematic. We agree with Craver that there is at least $a$ sense of explanation which is ontic: that real worldly mechanisms explain their phenomena by producing them. (Craver $2007 \mathrm{pp} 27-8$.) We do not deny that there is also an epistemic sense of explanation, such that the description of the mechanism explains the phenomenon. (Bechtel 2008 p16.) This debate is interesting on its own terms, but the difference is not as important to our project as it might appear. This is because even epistemic explanation in terms of mechanism description is still parasitic on the actual existence of mechanisms. ${ }^{3}$ Whether you think the explanation is i) the mechanism (ontic) or ii) the description (epistemic) you can still think the scientific process of mechanism discovery finds mechanisms. So both Craver and Bechtel can agree with us when we say that examining our practices of mechanistic explanation in these two fields tells us both about mechanistic explanation, and about mechanisms themselves.

We do not have space here to argue that there are no other important elements of mechanistic explanation, or of mechanisms themselves. There may be other ways in which protein synthesis and natural selection are different. In particular, Skipper and Millstein's arguments about productive continuity have yet to be addressed. This is of more concern to defenders of MDC's account than to defenders of the new mechanistic philosophy in general. Finally, although we hope to lay a useful groundwork here, further work will be necessary to see whether the three elements we do address apply to mechanistic explanation in all fields.

We will examine both protein synthesis and natural selection extensively in this paper, but we begin with an initial comparison. Protein synthesis is a paradigmatic mechanism. It is the mechanism by which cells make proteins, allowing them to survive, grow, and

\footnotetext{
${ }^{3}$ See our (2010) for further discussion. Bechtel has explicitly acknowledged this in private communication. See also Glennan 1996 p52.
} 
reproduce. It usually has three stages: replication, copying the cell's DNA; transcription, making RNA from DNA; and translation, making protein chains according to the RNA code. We know a great deal about these stages, including how the processes differ in different cells, what different types of RNA do, and what the code is - what protein a strand of RNA or DNA will produce. We have an in-depth understanding of this widelyfound mechanism.

Natural selection is an important mechanism of evolutionary change, classically conceived of as operating on populations - usually populations of organisms. Organisms vary in certain traits. Some traits increase the likelihood that organisms with these traits will survive and reproduce. If these traits are heritable, traits that are beneficial to survival and reproduction will spread through the population. If the environment remains relatively stable, the population will come to be better adapted to that environment. This is natural selection producing adaptation.

Natural selection, like protein synthesis, is called a 'mechanism', and regarded as explanatory. ${ }^{4}$ Evolutionary biologist Graham Bell writes: 'The main purpose of evolutionary biology is to provide a rational explanation for the extraordinarily complex and intricate organization of living things. To explain means to identify a mechanism that causes evolution and to demonstrate the consequences of its operation.' (Bell 2008 p1. $)^{5}$ If natural selection and protein synthesis are both explanatory mechanisms, one might assume they have something in common.

Experimental work does show some similarity between natural selection and protein synthesis. Both fields use manipulation experiments, as do many scientific studies. ${ }^{6}$ But apart from this, there are many differences. Detailed investigation of microstructure is crucial to protein synthesis. Since Franklin's x-ray diffraction photographs of DNA, breakthroughs have often been precipitated by new techniques for seeing molecular structure. ${ }^{7}$ These kinds of investigation are rare in evolutionary biology. On the other hand, artificial selection experiments, using multiple selection pressures on a single trait, are important in evolutionary biology. ${ }^{8}$ Such experiments are absent from protein synthesis.

\footnotetext{
${ }^{4}$ Both protein synthesis and natural selection are also often referred to as 'processes', sometimes in the very same paragraph in which they are called 'mechanisms'. This is initially puzzling, but is in fact a general phenomenon. When either are merely background factors in the context of explaining something else, they are called 'processes'. When their functions are salient, then they are called 'mechanisms'. See S2 for discussion of function.

${ }^{5}$ Skipper and Millstein agree: 'We think there is no question that contemporary evolutionary biology exemplifies the view that natural selection is a mechanism.' (Skipper \& Millstein 2005 p328-9.)

${ }^{6}$ Crick and Brenner's 1961 work using chemical mutagens to crack the genetic code is an important example. See Voet \& Voet pp1287-8. In evolutionary biology, for example, there is extensive use of experimental manipulations of clutch size to study whether clutch size is optimized. See Price's useful survey.

${ }^{7}$ See Bechtel 2006 for detailed examination of developing experimental techniques in the formation of the discipline of cell biology.

${ }^{8}$ See Bell 2008 p191-5 for description of a classic experiment carried out by Clayton and Robertson in 1957 on bristle number in Drosophila.
} 
This experimental work also advances the fields in different ways. Work in protein synthesis using breakthrough new technology, such as Franklin's photographs, require confirmation by a separate research group. But they are not otherwise repeated extensively. If the experimental work is of good quality, reproduction of results is relatively straightforward. This is in stark contrast to natural selection experiments, where replicate selection lines - further lines with the same intensity of selection - are standard. Some unexpected result or other is common, and experiments must be repeated many times to get solid results. ${ }^{9}$ It seems that the mechanisms of protein synthesis are generally more predictable than those of natural selection.

Perhaps because of this, the theory of natural selection itself is more mathematical, and dependent on the concept of probability: the correlation between having a trait and reproductive success, and the correlation between an organism having a trait and its offspring having that trait, are both important. Statistical modelling and simulation is common, and explanations often rely on graphs, equations and tables of data. Protein synthesis is different, with a focus on the structures and interactions of molecules, and explanations primarily involving diagrams of these structures and interactions. So one field relies heavily on mathematical description, the other on structural diagrams.

So these fields do differ, despite both natural selection and protein synthesis being called mechanisms. This theoretical concern about mechanistic explanation in general is rendered more urgent because sometimes both natural selection and protein synthesis are built into a single multifield mechanistic explanation. Presumably, this is more difficult if the very structure of mechanistic explanation used in the two fields is different. ${ }^{10}$ With this in mind, we will illustrate our more detailed points using just such a multilevel, multifield mechanistic explanation. We will use a survey paper by Janis Faye Hutchinson which explains: 'HIV may continue to be virulent because of its fast mutation rate, recombinogenic effect, and its use of human defenses to replicate itself. For instance, superinfection by viruses of different lineages has the potential for generating recombinant viruses with considerable genetic complexity. Such recombination could occur in humans to produce, for example, HIV-3 because biological mechanisms that usually constrain the evolution of viruses may not apply to HIV. That is, HIV may be evolutionarily free of constraints that could reduce its virulence.' (Hutchinson 2001 p99.)

\footnotetext{
${ }^{9}$ See Bell 2008 p193-4 for discussion of the unexpected results in Clayton and Robertson's 1957 work.

${ }^{10}$ Using elements from more than one field in a single mechanism is not unusual. Multilevel multifield mechanisms are very important to explain many phenomena. See Craver 2007, Russo 2008. We will come to the details of Skipper and Millstein's views in S4, but for them natural selection doesn't show parts anything like the parts of protein synthesis. If they are right, the difference between the two fields is significant enough to suggest a serious problem building both into a single mechanism.
} 
HIV in human population, body and cell
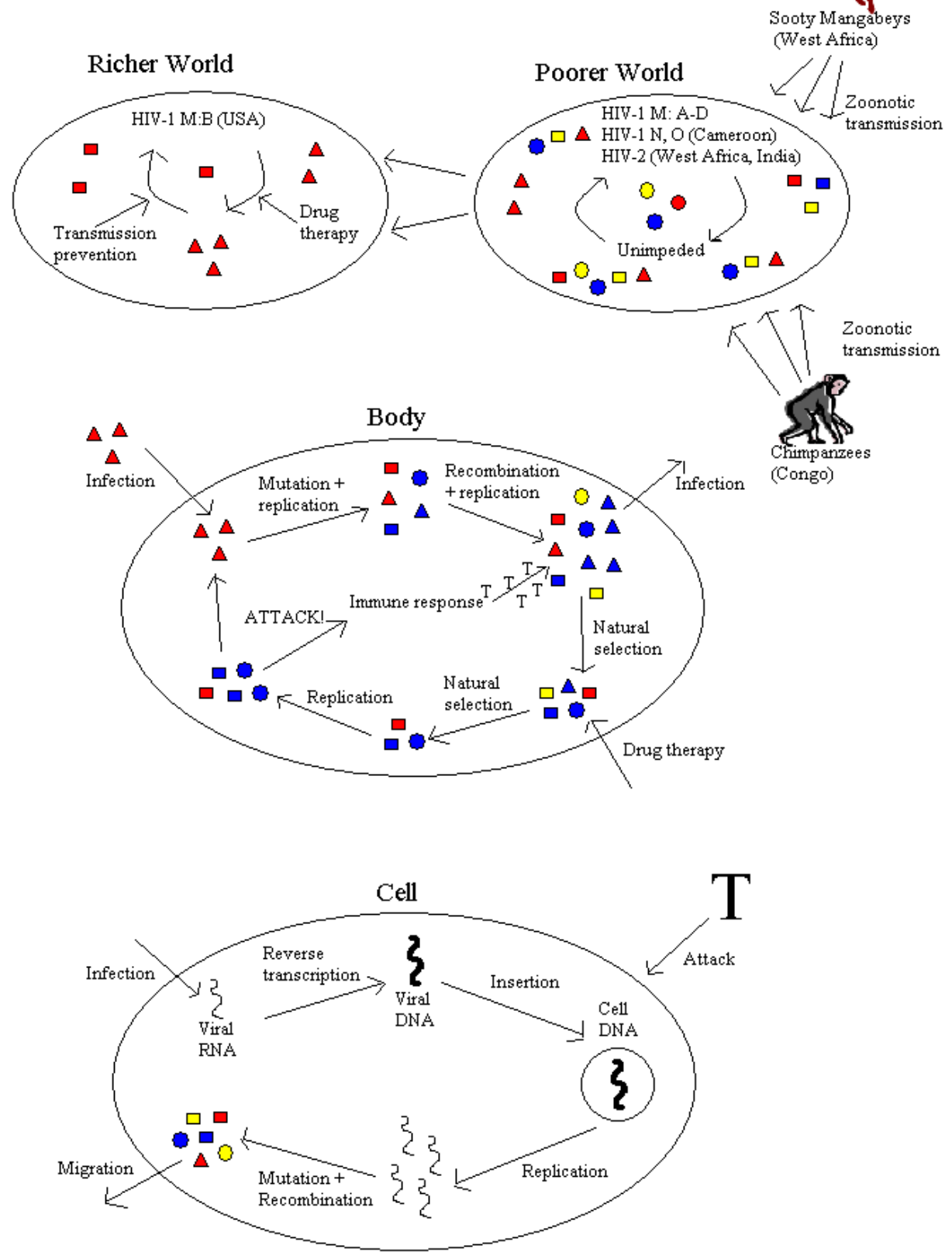

Hutchinson's explanation can be summarized in the diagram. The diagram is a simplification of what Hutchinson's paper describes, and the paper itself is a survey of more detailed work. Very briefly, the diagram illustrates that HIV is a problem for three main reasons. 
First, at the level of the cell, the HIV virus is so simple that it can be highly mutagenic and recombinogenic without losing functionality. Thus, the virus can change so that there are a variety even within a single host cell. Nevertheless, it can still do so much because it is a retrovirus. Its genome is RNA, which it reverse transcribes to DNA, which it inserts into a host cell's genome. From then on it can take over a large part of the protein synthesis apparatus of its now permanently infected host cell, creating many more viruses, which will not all be the same. Second, at the level of the human body, HIV directly attacks and disables our key defence mechanism - the immune system. This means that the HIV virus creates a favourable body for itself, while its sheer adaptability generates multiple strains even in a single body. This allows selection to favour the most virulent strains quickly, dampening all our efforts to combat the virus, at all levels: whether by the immune system, drug therapy, or vaccination. All these target a particular strain of HIV, and are not as effective against other strains.

Third, while we have some measures that do prevent transmission, these require a certain level of wealth and education, and so are effective only in the richer world such as Europe and North America. HIV is thought to have been transmitted in multiple zoonotic transmissions to humans from primate lentiviruses. There are two major strains: HIV -1 thought to have been transmitted from chimpanzees in the Congo; and HIV-2 thought to have come from the primate lentivirus SIV in sooty mangabeys in West Africa. HIV-2 is more closely related to SIV than it is to HIV-1. Both major strains of HIV have subtypes denoted by letters, most prevalent in particular geographic locations. Only HIV-1, subtype M, infecting the richer world, is receiving serious research. In the poorer world, particularly Africa, the many strains of HIV present are spreading virtually unimpeded, and recombining using already diverse strains to create potentially more deadly strains. There may be further zoonotic transmissions. There is a serious risk of a more dangerous major strain HIV-3 arising at some point.

The explanation here uses work from different fields, and the mechanisms of protein synthesis, and of natural selection operating on cellular, individual, and population levels. It is important to understand how the structure of mechanistic explanation allows work from these two different fields to be put into a common multilevel multifield mechanistic explanation.

We turn now to take functional individuation, decomposition and organization in turn in S2, S3 and S4, arguing that natural selection does satisfy each of these key elements when they are understood appropriately.

\section{Functional individuation}

Mechanistic explanation begins with the identification of the phenomenon to be explained, and the mechanisms discovered are partially individuated by the phenomenon they are responsible for. Note that mechanisms are also individuated in other ways. Two different mechanisms that produce the same phenomenon will still be differentiated on the grounds of different parts, and their organization, as we will discuss. Nevertheless, 
the identification of the phenomenon to be explained is a crucial starting point. Studying protein synthesis begins with a wish to understand how proteins are made, and protein synthesis is the mechanism for the production of proteins. In biochemistry textbooks, protein synthesis is understood as having the function of decoding the information in DNA to produce proteins. (See for example Adams et al. 1992, Voet \& Voet 2004, especially p92, and Whitford 2005, especially p247.) The lower-level mechanisms involved are also functionally described, deriving their functions from the function of the mechanism. For example, many repair mechanisms are understood as preventing or reversing malfunction.

Few philosophers writing on mechanisms have failed to notice this element of mechanistic explanation. For example, MDC write: 'Mechanisms are identified and individuated by the activities and entities that constitute them, by their start and finish conditions, and by their functional roles.' (MDC 2000 p6.) ${ }^{11}$

Not just any characterization of 'function' will do to understand the importance of function to mechanistic explanation. There are three senses of 'function' relevant to understanding mechanisms. We will take the classic example of the function of the heart to illustrate. The first is 'selected-effects' functions (see Wright 1973, Neander 1991 and Millikan 1984, 1993), where the selected-effects function of the heart is to pump, since it has been selected for on the basis of its pumping abilities. The second is Cummins' 'role-functions' (1975), where the role-function of the heart depends on the context in which we consider it. In the circulatory system, its role-function is to pump. When a newborn baby's head is laid on its mother's chest to calm it, the role-function of the heart is to make a familiar thump-thump sound. The third is Craver's 'isolated descriptions' (2001) which we prefer to call 'characteristic activity'. The heart has many isolated descriptions, but the one that is relevant to its selected-effects and role-functions is the heart's ability to contract. We will discuss these in turn, and see that Cummins' rolefunction is the most important for understanding mechanisms.

'Selected-effects' is probably the best known analysis of 'function'. Broadly, this is the view that the function of an entity is whatever it does that has been selected for in its evolutionary history, thus explaining the presence of the entity. To take the favourite example, the selected-effects function of the heart is to pump blood, since its bloodpumping ability has led to its being favoured by selection. In the histories of populations of animals with hearts, those with good heart-pumping have reproduced faster. The heart's noise-making ability, on the other hand, has been irrelevant to the reproductive success of animals with hearts. It has not been selected for, and so is not a selectedeffects function of the heart.

Cummins' role-function makes no use of selective history. For Cummins, the function of an entity is the role it plays in the overall behavior of a containing system. He writes: $x$

\footnotetext{
${ }^{11}$ See also Glennan 1996 p52, 2002 pS344. For further discussion, Darden and Craver (2002) usefully discuss some of the implications of the functional individuation of mechanisms, emphasising that an accurate description of the phenomenon is vital to the success of mechanistic explanation. Skipper \& Millstein agree that natural selection is like a mechanism in this sense (2005 p336).
} 
functions as a $\phi$ in $s$ (or: the function of $x$ in $s$ is to $\phi$ ) relative to an analytical account $A$ of $s$ 's capacity to $\psi$ just in case $x$ is capable of $\phi$-ing in $s$ and $A$ appropriately and adequately accounts for $s$ 's capacity to $\psi$ by, in part, appealing to the capacity of $x$ to $\phi$ in s.' (Cummins 1975 p762.) To return to the heart, if the overall capacity of the circulatory system in mind is to move food, oxygen and waste products around the body, then pumping is the role-function of the heart. This is because it is the heart's pumping that helps to explain this overall capacity of the circulatory system. We take this to be equivalent in a mechanism to Craver's 'contextual description'. Craver writes: 'A contextual description of some X's $\phi$-ing characterizes its mechanistic role; it describes X (and its $\phi$-ing) in terms of its contribution to a higher $(+1)$ level mechanism. The description includes reference not just to $X$ (and its $\phi$-ing) but also to $X$ 's place in the organization of S's $\psi$-ing'. (2001, p61.) This view of function is also well known. Indeed, it is becoming the dominant view in the mechanisms literature, for reasons that will become clear. ${ }^{12}$

Craver's isolated descriptions are less well known. He argues that they need to be recognised to defuse an ambiguity in Cummins' account as above. Craver writes: 'But this leaves it ambiguous whether the function is the capacity, described in isolation and simply "picked out" by its contextual role, or, instead, the contextual role by virtue of which the capacity is picked out. A complete description of an item's role would describe each of these. ... There is a difference, after all, between knowing that spark plugs produce sparks and knowing how that sparking is situated within the complex mechanisms of an engine.' (2001 p65.) The pumping of the heart can be picked out by its role-function or contextual description - its pumping as contribution to the capacity of the circulatory system. But, Craver is saying here, the pumping of the heart can also be picked out in a far more isolated way. The heart contracts. That is something we can recognize it doing regardless of context. That the heart beats was known, after all, long before the circulation of the blood was understood. Contracting in this way is a characteristic activity of the heart.

For mechanistic explanation, role-functions and isolated descriptions are both important. They are important both for understanding the function of a whole mechanism, regardless of context, and for understanding the function of components within a given mechanism. Selected-effects functions are relatively unimportant for either of these.

As we have said, mechanisms are mechanisms for a phenomenon. In that sense, mechanisms have functions. Selected-effects function does not help us understand this. The mechanism of protein synthesis exists in organisms with clear evolutionary histories, and so may well have a selected-effects function. However, natural selection itself has presumably not been naturally selected, and so can have no selective-effects function. ${ }^{13}$ But mechanisms in general require no naturally selected function. To get this clear, consider what happens when physicists examine mechanisms for the production of

\footnotetext{
${ }^{12}$ Bechtel endorsed it in person in a recent seminar, while MDC write: 'Functions are the roles played by entities and activities in a mechanism.' (MDC 2000 p6.)

${ }^{13}$ There is also a problem giving an account of malfunction on selected-effects views. See Davies. On that tricky issue, we follow Craver 2001 p72.
} 
gravitational waves. When massive bodies - stars and galaxies - move in appropriate ways, they will in theory emit gravitational waves, which makes particular kinds of movements mechanisms for the production of gravitational waves. There is no need for either an agent or natural selection to confer a this kind of function.

The relevant sense in which a mechanism as a whole has a function depends on whether the mechanism is situated in a context, or considered alone. If it is situated in a context, then it can have a role-function. The mechanism for the pumping of the heart, for example, might itself have a role-function. This is so if that mechanism is to be understood within the context of explaining the circulation of the blood. That itself might further be understood in the context of keeping the animal alive, and so on. But if there is no reference to a containing system, a mechanism has only an isolated description, or characteristic activity. This is where functional descriptions top-out. Animals try to stay alive. This is a characteristic activity of animals. We may or may not consider this as situated in a further context. Massive bodies engaging in certain movements produce gravitational waves. There need be no further system to which they contribute. 'Staying alive' or 'producing gravitational waves' are good candidates for isolated descriptions or characteristic activities of animals, or of some massive bodies.

So the least that mechanistic explanation requires is an isolated description of the mechanism - which is just a description of the phenomenon to be explained. Neither a further role-function nor a selected-effects function is necessary. As Craver says: 'Isolated descriptions of an X's $\phi$-ing specify the activity for which a lower-level mechanism will be sought and so fix the active, spatial, and temporal boundaries of that mechanism ...' (2001 p65.) Natural selection having an isolated description is no problem. Natural selection explains adaptation, because natural selection characteristically produces adaptation. So natural selection is a mechanism for adaptation.

It might be thought that characteristic activity is not really an account of function. The claim about language is not crucial for us here. The important claim is that natural selection fulfils what is necessary to count as a mechanism. Nevertheless, we have two main reasons for counting it as such. First, it captures the barest sense in which a mechanisms is a mechanism for a phenomenon. Secondly, it fits with the language of the functional individuation of mechanisms - the individuation of mechanisms in terms of what they $d o$. Characteristic activities are the barest account of what that demands. ${ }^{14}$

Our HIV example illustrates the interplay of the three senses of function. The explanation is structured by the conception of the phenomenon - the difficulty of treating and containing HIV. This can be treated either as a bare isolated description, or within various social and epidemiological contexts as a role-function. Because the domain involves selection pressures at multiple levels, many of the functions here might well be naturally selected. But it is what the elements of the mechanism actually $d o$ that is crucial. Now that the mechanism is set, a context is given, so these are role-functions.

\footnotetext{
${ }^{14}$ It is also one legitimate interpretation of the original Aristotelian use of ergon, so it also has historical antecedents in theorizing about function.
} 
The gag gene is important to HIV, allowing the virus to migrate out of the host cell. If a mutation renders it non-functional, it stops the virus. There are two points to notice. The first is that it might be hard to come by evidence that the gag gene has itself been naturally selected, as opposed to being fixed by hitchhiking, or by drift. ${ }^{15}$ The second is that the role of the non-functional gene, stopping HIV from migrating, presumably has not been naturally selected. Yet what it does in stopping the virus from migrating is important. In both cases, it is what the gene actually does, not its selective history, that matters. These are Cummins' role-functions. Clearly, if we use only naturally selected functions, there won't be enough functions for all the function-ascriptions we use in mechanistic explanation. This is because we will not be able to ascribe a function to many such entities as the non-functional gag gene.

In conclusion, the mechanisms of protein synthesis and natural selection are both functionally individuated. They have at the least characteristic activities, and if they are situated in a context, they might also have role-functions. We will show in S3 and S4 that function is a more important element of mechanistic explanation than might at first appear, since it is crucial to correctly understanding both decomposition and organization. In these sections, role-functions will be the most important sense of function.

\section{Decomposition and mechanistic hierarchy}

Once the phenomenon is identified, mechanistic explanation characteristically proceeds by decomposing the phenomenon into lower-level components. The activities of lowerlevel components are often regarded as further phenomena and further explanations sought, so that decomposition moves to components another level down. This may iterate many times. So mechanisms discovered are usually located in just such a nested hierarchy, with relations to both lower-level and higher-level mechanisms in the hierarchy. Indeed, as we have said, identifying the phenomenon might well involve situating it with regard to higher-level mechanisms. This would give the mechanism for the phenomenon a role-function, rather than merely an isolated description.

Decomposition is uncontroversial in the philosophical literature. MDC write: 'Mechanisms are entities and activities organized such that they are productive of regular changes from start or set-up to finish or termination conditions.' (MDC 2000 p3.) Bechtel and Abrahamsen's favoured characterization is: 'A mechanism is a structure performing a function in virtue of its component parts, component operations, and their organization.' (Bechtel and Abrahamsen 2005 p423.) ${ }^{16}$ Notice that decomposition is into two kinds of parts. The language varies, but there are always bits of the mechanism ('entities' or 'component parts'), and something these bits do ('activities' or 'component

\footnotetext{
${ }^{15}$ Philosophers have a tendency to assume something has a historically naturally selected function just because it exists in something which has a clear evolutionary history - such as an organism. We thank William Bechtel for pointing out in a recent seminar that very few such assumptions are sufficiently supported by empirical evidence of a history of selection for that particular trait.

${ }^{16}$ See also MDC 2000 p13 on nested hierarchy, and Bechtel \& Abrahamsen 2008 p560 on decomposition.
} 
operations'). We will use MDC's language of activities and entities for clarity, but mean only to mark this uncontroversial distinction. ${ }^{17}$

This decomposition is clear in protein synthesis. Consider a list of commonly used expressions from three biochemistry textbooks (Adams et al, Voet and Voet, Whitford). Entities: DNA, RNA, ribosome, operator, enhancer, promoter, RNA polymerases, repressor, activator, Watson-Crick base pair, covalent bond, replication fork, codon, anticodon.

Structures: Loop, cleft, lobes, two-stranded, helical, ring, backbone, hairpin, wound, segment, position on gene, wrapped, elongation, strand, 'hairpin' or stem-loop structure, globular or dumbbell shape, helix-loop-helix structure, chain.

Activities: Replicating, transcribing, translating, trigger, binding, phosphorylates, modifying, wrapping, folding, cutting, catalyse, protect, opening, unwinding, supercoiling, breaking, inhibiting, stabilizing.

The language used to describe entities, their physical structure and activities is amazingly rich. These kinds of components allow detailed description of the sub-mechanisms of protein synthesis to see how DNA is decoded to produce protein. But as we have said, this kind of concern with physical parts and their microstructure is apparently absent from natural selection. Perhaps natural selection is not decompositional.

In the rest of S3 we will examine arguments against the decomposition of natural selection (S3.1). We will argue that decomposition is functional decomposition even in protein synthesis, since successful structural decomposition is into functionally relevant parts (S3.2), identify some of the entities and activities of natural selection, and finally respond to the arguments against the decomposition of natural selection (S3.3).

\subsection{Arguments against the decomposition of natural selection}

Skipper and Millstein (2005) argue that natural selection does not have the right kind of parts to be decompositional. They take Glennan's (2002) and MDC's views of mechanisms in turn. They don't think natural selection has parts fitting Glennan's characterisation: '[Glennan] most recently ... says that the properties of a part must be stable in the absence of interventions, or, as he subsequently clarifies, the complex of objects must form a stable enough configuration to itself be called an object.' (Skipper and Millstein 2005 p336.) Skipper and Millstein examine in turn the possibilities of organisms, the environment, and populations being the parts of natural selection. They allow organisms as a part, but say organisms cannot be the only parts of natural selection in the full story. They object to the environment as a part, since it might be many parts, and may not have stable enough properties to satisfy Glennan's criterion. Populations, similarly, do not have stable enough properties. ${ }^{18}$

\footnotetext{
${ }^{17}$ We deal with more controversial aspects of MDC's entity-activity dualism elsewhere (forthcoming).

${ }^{18}$ Skipper and Millstein also object to taking populations as an entity in a mechanism that changes that population (2005 p337). Barros comments: 'Skipper and Millstein did not provide any elaboration on this point, and it is far from clear why as a general matter it is problematic for an entity to be part of a
} 
In a recent paper, Glennan expands on this problem. He writes: 'Entities need to be localized in space and time; they need to engage in particular activities at particular times and places. The population in the water bug case does not have these properties. The population as a whole is spread out and does not engage in collective activities. ... What makes a collection of parts into a single entity is that these parts have a stable structure, that the stable structure engages in activities as a unified entity, and that these collected parts share a common fate. ...One cannot say categorically that populations either are or are not individual entities.' (Glennan forthcoming p9 See also Glennan 1996 p53.)

Skipper and Millstein's argument moves on to MDC's view, that the components of mechanisms are activities and entities. Skipper and Millstein claim that while activities and entities can be found that are relevant to natural selection, they are not components of natural selection. Again, Skipper and Millstein allow organisms such as moths to be parts - in this case MDC's 'entities'. But then, they say, the relevant activities must be things the moths do like feeding and mating. However, this is not good enough: 'The problem is that the activities of surviving and reproducing do not constitute selection. Differently put, the ways in which the finches and moths engage in survival and reproductive activities are elemental of selection. But just enumerating those entities and activities does not result in an articulation of the mechanism of natural selection.' (Skipper and Millstein 2005 p341.) The thought is that although organisms must feed and mate and die for natural selection to happen, such activities are not part of the mechanism of natural selection itself. This is a subtle and important point. Natural selection is supposed to explain what happens to a population as a whole, while listing the activities of the individual organisms will: 'result in an articulation only of the life history of a population: Organisms are born, they eat, they mate, they die.' (Skipper and Millstein 2005 p341.)

By way of conclusion Skipper and Millstein write: 'The straightforward way to articulate natural selection as a mechanism is by explicating all of the stages integral to causing populations to change. The stages themselves are not parts in Glennan's sense or entities in MDC's sense. They are, instead, salient, if not idealized, time-slices of the process of natural selection.' (Skipper and Millstein 2005 p339.) These time-slices are the only parts that they can find.

There are serious obstacles to understanding natural selection as a decomposable mechanism. The absence of detailed investigation of microstructure in natural selection is no accident. Other than individual organisms, there are no parts like the parts in protein synthesis, and the natural selection story cannot be told with a list of things happening to individual organisms. As parts, populations certainly don't have the stable structures that, for example, a DNA molecule or a protein does. And precise spatial location or orientation of parts, a core concern in protein synthesis, is far less important in natural selection. Glennan and Skipper and Millstein have identified some genuine 
worries. If these stand up, it is puzzling how we fit both protein synthesis and natural selection in a single mechanistic explanation.

\subsection{Decomposition is functional decomposition}

Before concluding that the two fields are radically different, it is worth a more careful examination of how explanation by decomposition works even for protein synthesis. The key thing to notice is that the obsession with physical structure in protein synthesis exists because such physical structure has been discovered to be of vital functional importance. It is functional decomposition that is vital.

The parts of protein synthesis are functionally individuated. Whitford explains: 'In many instances determining structure has uncovered intricate details of their biological function.' (Whitford 2005 p310.) The function of the components of a mechanism, remember, are Cummins' role-functions. The function of an entity is the role it plays in the overall behaviour of the mechanism, so the function of an entity derives from the function of the mechanism as a whole. Operators, enhancers, RNA polymerases, codons and so on are categorised and named in terms of what they do in the job of decoding DNA and producing protein. Entities are grouped according to the properties they have in common that are relevant to the operation of the mechanism - their role-functions. For protein synthesis, these relevant properties are often structural. ${ }^{19}$

Structure is described even when functional relevance is not immediately apparent, partly because it might be revealed as relevant later. Biochemists are also aware that objects with similar functions don't always have similar structures. AaRSs (aminoacyl-tRNA synthetases) are enzymes which stick the right amino acid on the tRNA. There are over 1000, and they don't have similar structures: even the aaRSs that stick the same amino acid to the tRNA are diverse. The relation between structure and function is a defeasible assumption, but much of the time structure and function are related. The association of structure and function is an empirical discovery crucial to progress in biochemistry.

The mechanisms literature view of decomposition recognises this. Bechtel and Abrahamsen contrast structural and functional decomposition, but they recognize that functional decomposition is more important: 'it is important to emphasize at the outset that the structural components into which researchers seek to decompose a system are ones which perform the operations that figure in the functional decomposition. The majority of ways of structurally decomposing a system will not result in components that perform operations.' (Bechtel and Abrahamsen 2005 p433.) ${ }^{20}$ Glennan is right that something cannot be an entity in a mechanism without some sort of stability, but it is functional stability - whether it has a role that contributes to the phenomenon - that is important.

\footnotetext{
${ }^{19}$ See for example the discussion of helix-loop-helix proteins in Adams et al., p 431.

${ }^{20}$ See also Craver 2007 p188, Glennan 2002 S344. See Bechtel 2006 for extended discussion of the importance of mapping structural information coming from cytology to functional information coming from biochemistry in the creation of the new discipline of cell biology.
} 
Entities are not the only kind of part - a mechanism needs activities (or component operations) too. Notice that in a classic mechanistic hierarchy, the distinction between an activity, or entity, and a mechanism, is a fluid one, relative to a level of description. What is an activity like catalysis, or an entity such as an enzyme at one level of description, at a lower level of description might be an entire multilevel mechanism. People are entities in many mechanisms, but there are countless biological, psychological and neurological mechanisms that make up people. Similarly for cells. This is widespread, so that something treated as an entity or activity at some level of description is in fact a mechanism at a lower level of description.

Activities are interesting because they often are the functions of the entities - an activity is what an entity does, or what two or more entities do together. Activities are individuated in a similar way to entities in the hierarchy of mechanisms. Activities are identified in terms of their contribution to the behaviour of the phenomenon to be explained. This is why a repair mechanism in protein synthesis is a mechanism for the reversal of malfunction. The roles of both entities and activities are devolved from the phenomenon being explained, so they both have role-functions. ${ }^{21}$

So the structural focus in protein synthesis is misleading. What is most important to decomposition in mechanistic explanation in general is functional decomposition. The mechanism must be decomposed into parts - entities or activities - that perform different functions, and mechanisms in other domains might not have parts that are physically similar to the kinds of parts which are functionally relevant in protein synthesis.

\subsection{Functional decomposition in natural selection}

We now have a better framework for re-considering the parts of natural selection. We will argue that the mechanism of natural selection does fit into a functional hierarchy one of mechanisms decomposed into sub-mechanisms. ${ }^{22}$ Once the hierarchy itself is clear, both entities and activities in the various mechanisms can be detected, individuated in terms of their roles in the production of the phenomenon. With this in place, we will then respond to the more specific worries raised by Skipper and Millstein.

\footnotetext{
${ }^{21}$ This is not to say that function and activity are the same. The function of an entity might, for example, be a capacity rather than an activity, such as in a mechanistic explanation of the fragility of glass. But activities and the role-functions of entities are closely related. Craver recognises this: 'The entities in mechanisms can be taken to correspond to Cummins' $\left\{X_{1}, X_{2}, \ldots, X_{m}\right\}$; they are the physical parts of the mechanism (e.g., the hearts, kidneys, and veins). Activities are the things that these entities do, either by themselves or in concert with other entities. The activities in mechanisms can be represented as the $\left\{\phi_{1}, \phi_{2}\right.$, $\left.\ldots, \phi_{\mathrm{n}}\right\}$ in Cummins' account.' (2001, p57.) See also: 'A contextual explanation explains an entity or activity by showing what it is for, that is, how it fits into the organization of a higher-level mechanism.' (2001, p70.)

${ }^{22}$ In this paper we are concerned with explanation rather than prediction. Predictive projects in natural selection might aim to model a population, using aggregate variables with no regard to the population's structure. But this is to ignore a wealth of explanatory detail.
} 
Before beginning, it is worth distinguishing between a mechanism like protein synthesis in the abstract, and in particular, concretely instantiated, cases. In the abstract, "protein synthesis' is a name for a bundle of related mechanisms that do similar jobs, but only have so much in common. (Darden 2006 chapter 4.) Mechanisms may be quite different in different organisms - consider the retrovirus HIV, which has RNA as its genetic material, and must reverse transcribe it to DNA, inserting it into the host cell genome. This is non-standard. Together, this bundle forms a large explanatory resource to understand what happens in particular kinds of cells, or particular histories of a kind of cell. But in the particular case, a particular mechanism operates.

We will argue that natural selection is the same. We will take the relatively abstract first, but show that to build an explanation for a particular population, the more abstract schemas must be gradually made increasingly concrete. In all cases, the parts are distinguished primarily by their role functions. Natural selection is commonly subdivided into three distinct kinds. The classic case is directional selection, when the population is not well fitted to the environment. The average character trait in the population will shift. The second case is stabilizing selection. When the environment is stable, extreme trait types are selected against, increasing the proportion of the population exhibiting the average trait. The third is disruptive selection, which occurs when extreme trait types are fitter than the average, and so increase in number, leading to a bimodal distribution. For example, certain species of lizard have diametrically opposed reactions to predators: they either freeze, or they run very fast. Run slowly, and you get eaten. These are all different ways of producing adaptation: adaptation to environmental change, to a constant environment, and to divergent selection pressure, each with characteristic effects on the distribution of traits in the population. They all have different roles in the overall production of adaptation.

Another important division reflects Skipper and Millstein's view that the only 'parts' of natural selection are phases, or time-slices. There are recognisable phases in what happens to a population when selection pressure is applied. The first is sorting, where organisms with worse traits die, and those with better traits reproduce faster. During sorting, the mean fitness of the population cannot exceed the fitness of the fittest type present before selection. In the second phase there is time for new variation to be generated by recombination - combining the DNA of two individuals in the DNA of a single individual, most familiarly by sexual reproduction - or mutation, and these new variations are also sorted. This phase still has limits, depending on how many loci on the DNA affect the character being selected, and on the size of the population. (See Bell 1997 p159.) The third phase is when adapted variants in the population undergoing these changes in the short-term increase. Note that ultimately medium-term constraints on the population are themselves subject to being changed by evolution. In the long-term, many things can happen, such as the emergence of new methods of reproduction, or multicellular life, or the divergence of two species. These phases are rough time-slices of directional selection. But each has a different role, producing sorting, new variation, and ultimately many other things. And just as for the time slices of protein synthesis replication, transcription and translation - the explanatory project does not stop there. 
Further sub-mechanisms are relevant. Not all of these are clearly part of selection, being at a much lower-level. But many sub-mechanisms creating variation, heritability of traits, and means of reproduction - different roles - structure the population and the selection process itself as it takes place. To think of these as part of natural selection is natural enough for Godfrey-Smith to write: 'It [this book] is primarily about evolution by natural selection, the process by which populations change through a dynamic of variation, inheritance, and reproduction.' (Godfrey-Smith 2009 vii.) It is also natural enough for Skipper and Millstein to include variation in natural selection, as their stage 1, and mutation as change in stage 1. (Skipper and Millstein 2005 p329-31.) At a slightly lower level than natural selection itself, frequency-dependent selection and sexual selection are important sub-mechanisms. At a much lower level, the detail of reproduction clearly helps explain how a population responds to environmental change. Evolutionary biologists also want to know about the structure of DNA, and morphogenesis. Gene linkage, pleiotropy and epistasis affect how adapted the organism can become. There are dozens of other relevant mechanisms, such as for example autoselection and meiosis as low-level mechanisms. Recombination and mutation are much-studied, with their sub-mechanisms and related mechanisms such as transduction, transformation and conjugation. As our understanding of cell mechanisms has improved, more evolutionary biologists look to the level of the cell to help understand the evolutionary history of populations. The use of molecular mechanisms and natural selection is very striking in our HIV example. The error-prone reverse transcriptase that creates DNA from the viral RNA is a molecular mechanism that generates many viral variants on which natural selection acts to screen HIV strains resistant to drug therapy, creating huge difficulties for making effective drugs. This kind of use of mechanisms on many different levels is no longer uncommon.

All of these mechanisms do different things, which contribute in different ways to the overall evolutionary trajectory of the population - the phenomenon to be explained. These mechanisms all have different role-functions. In understanding all of these, evolutionary biologists have a deep understanding of the many detailed ways how populations evolve, in response to subtle differences in kind of selection pressure, and in the available responses of that population.

So natural selection even in the abstract does display some kind of parts - most clearly, it has sub-mechanisms. Remember that the activity-mechanism distinction is fluid, so that a mechanism at one level of description is an activity in a higher-level mechanism. The sub-mechanisms we have identified are all also activities. With the activities clear, it is easier also to identify the entities. With this work done, we can also respond to Skipper and Millstein's failure to identify entities and activities in natural selection, by looking at this hierarchy. We suggest the following are possible components of natural selection: Activities: Directional selection, stabilizing selection, disruptive selection, sorting, sexual selection, frequency-dependent selection, recombination, reproduction, meiosis, epistasis. Entities: Populations, organisms, cells, DNA, chromosomes, alleles.

The HIV example illustrates how these explanatory resources are drawn on, to allow multilevel multifield mechanistic explanations. Hierarchical relations are only implicit in 
Hutchinson's paper, but there are at least three important levels - the cell, the body, and the human population. Activity is very important. Entities engaged in activities, such as RNA strands recombining, produce phenomena, such as a new HIV strain with new properties, that matter at higher levels such as the effect of the activity of the HIV on the immune system of an individual, drug development for the country, and international policies for containing HIV. Natural selection operates in all these cases, and both entities and activities are described in terms of their contribution to the overall phenomenon.

Moving now to the simple concrete cases, it is even easier to see that there are both entities and activities in natural selection. ${ }^{23}$ We can also see that these parts of natural selection are spatiotemporally located, structured, and stable enough to produce adaptation - the phenomenon they are invoked to explain. We can reply now to Skipper and Millstein's claim that in natural selection there are no entities - other than organisms - sufficiently stable and localizable to count as parts. Along with Glennan, they deny that populations are entities in natural selection. We think they are.

Selection processes happen in a population or organism that is at a particular place at a particular time. It is true that the precise spatial location and particular orientation of the population or organism seldom matters, but this does not mean that the population isn't spatiotemporally distributed at all! Consider the selection pressures operating on HIV-1, subspecies M: A. This is a widely distributed population, but it is spatiotemporally located - it's in Africa, now. And this matters - that strain is getting far less research and investment than the subspecies M: B infecting the richer North America and Europe.

Particular populations and organisms will have structures that matter to natural selection. For example, a lower effective size of population can make a large population respond to selection pressure as if it were a small one, as can breeding cycles that involve bottlenecks. The structure of replication, and any gene linkage or interaction matter too for both organisms and populations. Bell comments: 'Spatial structure may modulate the effect of selection, even if an allele has the same fitness in every deme. Selection in a large unstructured population acts exclusively through differences in fitness among individuals. In a subdivided population selection will act in the same way within each deme.' (Bell 2008 p156.) Structure of entities matters to natural selection. It does not matter as often as structure matters in protein synthesis, but it does matter.

We agree with Glennan, and Skipper and Millstein, that some kind of stability of parts is needed in a mechanism. However, the extent of stability required can be exaggerated for both protein synthesis and natural selection. The entity only needs to be around, or the activity endure, for long enough to contribute its role to the overall phenomenon. Important changes in either bacterial or viral populations might be very brief, but if they are functionally relevant, in that they do something that contributes to the overall phenomenon, they need to go in the explanation. Protein synthesis has similarly fleeting

\footnotetext{
${ }^{23}$ Barros uses a particular example of crab predation on snails to argue that natural selection is a two-level mechanism. See Barros 2008. We take our work to be an extension of Barros'. Clayton and Robertson's 1957 work on Drosophila is also a nice simple concrete case for natural selection. See Bell 2008 p191-5.
} 
entities: RNA is made from DNA, used to make protein, and broken down again almost immediately. The entities and activities of natural selection are stable enough to stick around and produce phenomena we can recognise, study, and explain. This kind of stability is all that is required. This makes them candidates to be entities and activities in a recognisable mechanistic explanation.

In the HIV mechanism, there are some fairly stable parts, such as human populations, infected human beings, and RNA in viral cells. But this stability is limited. RNA creates many different strains, and the virus works by reverse transcribing RNA to DNA and inserting DNA into the host genome. Further up, HIV infection works by changing the host body - disabling its immune system. HIV can change a population too: consider how populations have altered their sexual practices. In all this, understanding the lowlevel mechanisms of mutation and recombination is vital to understanding the responses of both individuals and populations. The recombination that eventually produces HIV -3 , and the conditions that allow it to spread, might be a very brief, local, unstable event, with far-reaching consequences. In all this, functional individuation is key. Even the most stable parts of the HIV mechanism are only important for what they do - such as the gag gene which allows the new viruses to migrate out of host cells.

So in natural selection there are entities and activities which are spatiotemporally located, and stable enough to be used in explanation. This includes populations. As GodfreySmith writes: 'A population is a physical object, bound by ancestry and other causal relations, internally variable at any time and changing over time.' (Godfrey-Smith 2009 p11.)

\subsection{Conclusion}

Mechanistic explanations do involve two kinds of parts: activities and entities. ${ }^{24}$ These are functionally individuated, in terms of their contribution to the phenomenon they are invoked to explain. It is a discovery of a discipline - even of a particular mechanism what the functionally relevant entities and activities are.

Protein synthesis and natural selection are analogous, in spite of the differences we picked out in S1. Often in natural selection the activities are more important than entities to the explanation of the phenomenon. The activities are different things that populations of relatively similar entities (such as organisms of the same species) can do. The entities must be there, of course, but we find many different activities - sub-mechanisms studied because these differences are more often functionally vital. In contrast in protein synthesis the structural differences between kinds of entities are more often vital. There is a greater concentration on the detail of microstructure in protein synthesis because microstructure is often of functional importance, and entities have functionally vital structural differences. Nevertheless, entities and activities are both needed in both mechanisms.

\footnotetext{
${ }^{24}$ Or component parts, and component operations, in the language of Bechtel and Abrahamsen. (For example, their 2005 p423.)
} 
In their conclusion, Skipper and Millstein comment: 'We cannot claim that the selection mechanism always has the same working entities (or the same kind). Thus, under the assumptions in force here, providing a univocal mechanism for natural selection on MDC's account is unworkable.' (Skipper and Millstein 2005 p341.) Skipper and Millstein are right that natural selection does not always have the same kind of working entities. Natural selection can be very different in different populations at different times - as can particular cases of protein synthesis. We agree with Skipper and Millstein that forcing similarity where there is none can be unhelpful. Nevertheless, we think the common understanding of mechanistic decomposition we have identified here is worth having - particularly for its ability to make sense of multifield mechanisms.

\section{Organization completes the explanation}

Identifying the entities and their activities is not enough to explain the phenomenon. You have to understand how they produce the phenomenon of interest together - how they are organized. The importance of organization in the production of phenomena, and so in our full understanding of the phenomenon, is uncontroversial. MDC write: 'The organization of these entities and activities determines the ways in which they produce the phenomenon.' (MDC 2000 p3. See also Bechtel and Abrahamsen 2005 p435, and independent work by Darden 2002 S355.)

Like decomposition, organization is easier to understand for protein synthesis than natural selection. Here we will examine Skipper and Millstein's arguments against the organization of natural selection (S4.1). We will argue that organization in general needs to be understood in terms of contribution to the phenomenon - whatever set of relations among the parts allow them to produce the phenomenon (S4.2) - and finally discuss forms of organization found in natural selection (S4.3).

\subsection{The Problem}

The entities and activities of protein synthesis are easy to identify and some aspects of their organization are clear. They produce proteins in concert by virtue of their temporal and spatial arrangement. In recent work, Craver explores this in some detail. He summarizes his view: 'By organization, I mean that the parts have spatial (location, size, shape, and motion), temporal (order, rate and duration), and active (for example, feedback) relations with one another by which they work together to do something. Organization is the interlevel relation between a mechanism as a whole and its components.' (Craver 2007 p189.) Several aspects of spatial organization can be important. Craver gives a non-exhaustive list of: sizes, orientations, conformations or shapes, locations, contact or connection. Temporal organization also matters. The order, rate and duration of successive component activities are crucial to what the mechanism does. 
These aspects of organization matter to protein synthesis, but it is not clear how to understand them for natural selection. Skipper and Millstein argue that natural selection does not involve organization. They write: 'The claim that mechanisms are organized implies (1) that mechanisms are comprised of various elements and (2) that those elements are structured in some way.' (2005 p336.) Since Skipper and Millstein hold that natural selection does not have the right kinds of parts, they cannot think the parts are structured. Nevertheless, they give positive reasons for worrying about organization in natural selection. They point out that organisms and populations do not need to do things in any particular location, can change in structure and orientation, and events need not have any particular temporal order. There is a certain amount of organization of organisms involved, they concede, but not enough to satisfy MDC's account. This means, they say, that there is no organization common to all episodes of natural selection. They write: 'In other words, we can give no general account of organization in populations undergoing natural selection. And if that is right, then there is no general mechanism, sensu MDC and Glennan, of natural selection to be found. ${ }^{25}$

Skipper and Millstein have identified genuine obstacles to thinking of natural selection as involving organization. In the next sub-section we will examine organization theoretically, arguing that many forms of organization are important in understanding mechanisms, but providing an account of what these forms of organization share. We will then move on in S4.3 to examine organization in natural selection and protein synthesis, arguing that when organization is understood correctly, it does apply to natural selection.

\subsection{What is organization?}

In this section, we address Skipper and Millstein's second and more overarching point that there is no general account of organization in natural selection. We examine organization in mechanisms from a theoretical point of view, and argue that there are many forms of organization that are important in understanding mechanisms and mechanistic explanation. We will provide an account of what these forms of organization have in common. But we hold that it is desirable that which particular forms of organization might be important for particular mechanisms is not fixed in advance. Which particular forms of organization contribute to the production of a particular phenomenon is an empirical discovery - a crucial part of mechanism discovery.

Skipper and Millstein write: 'It is puzzling to speak of the arrangement of and connections between non-physical parts.' (Skipper and Millstein 2005 p136.) But spatial and temporal organization are not the only forms of organization. Consider a simple kind of organization - the part-whole relation. Craver himself points out that one's holdings in a particular bank account are part of one's total wealth, but this part-whole relation is not spatial. Nor is it temporal. So even simple forms of organization are not merely

\footnotetext{
${ }^{25}$ Skipper and Millstein 2005 p338-9, emphases in original. Skipper and Millstein offer their positive account of the closest thing to organization in natural selection p339, but it is incidental to our arguments here.
} 
spatiotemporal. ${ }^{26}$ Organization more generally is merely some feature or other of the components. Mitchell writes: 'Complex systems are also characterized by the ways in which the parts are arranged, that is, the relations in which the components stand or their structure.' (Mitchell 2003 p167.)

Not just any features of entities and activities will give you the organization of a mechanism. Organization is the final element in the explanation. We identify a phenomenon to be explained, and discover the entities and activities relevant to that phenomenon. Finally we discover what kinds of relations the entities and activities stand in that produces that phenomenon. We are only interested in those features. Activities and entities aren't organized in the relevant sense just because there are relations among discrete identifiable bits, but only if the activities and entities each do something and do something together to produce the phenomenon. ${ }^{27}$ There is no reason to suppose that the features by which a mechanism produces one kind of phenomenon will be the same as those by which a different mechanism produces a different kind of phenomenon. What particular kinds of features of the parts produce the phenomenon is an empirical discovery of the domain, and the mechanisms there. If this is so, then it is an empirical question whether all forms of organization are merely spatiotemporal. However the parts interact to produce the phenomenon, whatever kinds of relations allow them to do this, that's what we're after to complete our explanation of the phenomenon.

Some philosophers recognise forms of organization beyond the merely spatiotemporal. Bechtel writes: 'One of the key features of organization in biological mechanisms is the incorporation of feedback and other kinds of control systems that allow the behaviour of some components of the mechanism to be regulated by other components of the mechanism.' (Bechtel 2006 p33.) Later Bechtel adds homeostatic mechanisms. Mitchell discusses self-organization, important in complex dynamics: 'self-organization processes are ones in which higher-level order emerges from the simple interactions of component parts in the absence of a pre-programmed blueprint.' (Mitchell 2003 p6.) Feedback, control systems, homeostatic mechanisms and self-organization are all layers of organization beyond the merely spatial and temporal. What matters for a homeostatic mechanism, for example, is not a spatiotemporally located trigger, but the fact that the system will be pushed back to its original state after any small perturbation. There are probably many more forms of organization still to be discovered and classified.

It is no great stretch for the rest of the mechanisms literature to recognise these forms of organization. Craver mentions feedback as an element of active organization. (Craver 2007 p189.) He might be read as holding that 'active organization' is the element of

\footnotetext{
${ }^{26}$ See Craver 2007 p186. All the forms of organization we will discuss are, presumably, realized by physical systems with identifiable spatio-temporal relations. We do not deny this. Nevertheless, we will argue, many of the forms of organization studied by the sciences are not best thought of as spatiotemporal.

${ }^{27}$ This captures the prevalent idea that organization is what allows the whole mechanism to do more than the sum of its parts. See Craver 2001 p59, Bechtel 2007 p191. We don't want to insist that in all mechanisms the whole does more than the sum of the parts. However, in many mechanisms, it does. Cummins recognizes the importance of organization to functional analysis (1975 p764). So do many biologists. See for example Laublicher and Wagner p58.
} 
organization that goes beyond the merely spatio-temporal, although it is at root realized by a spatiotemporally located system.

At a certain level of abstraction, Skipper and Millstein are right. We could not say that all mechanisms will show, say, negative feedback loops as a vital form of organization.

But at a different level of abstraction there is something in general that we can understand about organization: it is whatever features exist by which the activities and entities each do something and do something together to produce the phenomenon. There are many interesting forms of organization that are not merely spatiotemporal, and there is no reason to suppose that different mechanisms will exhibit precisely the same forms of organization. The forms will be further illustrated with regard to both natural selection and protein synthesis in the next section.

\subsection{Organization in natural selection and protein synthesis}

In this section we will illustrate the theoretical arguments of the previous section by examining organization in protein synthesis and natural selection. We will also address Skipper and Millstein's first point, arguing that natural selection does show spatiotemporal organization. In comparing organization in the two domains, we will further argue that the difference between them is not so great as it appears. Natural selection and protein synthesis both show both simpler and more complex forms of organization.

Skipper and Millstein's first objection to organization in natural selection is that organisms and populations do not need to do things in any particular location, can change in structure and orientation, and events need not have any particular temporal order. But consider again the distinction we made above between natural selection as a highly abstract bundle of mechanism schemas, and natural selection as it operates in a particular, concrete, spatiotemporally located population.

We argued above that in the particular case, we can identify spatiotemporally located and structured entities. Just as in that case, we can identify when organization is important to natural selection. Indeed, we already identified forms of spatiotemporal organization that matter, such as the structure of a population undergoing natural selection. Skipper and Millstein are quite right that very precise locations and changes in orientation do not often matter to natural selection, as they do in protein synthesis. Nevertheless, these things do matter. As we have said, the spatiotemporal organization of various strains of HIV matter a great deal to which strains are being successfully controlled. HIV-1 subspecies M: B affecting North America and Europe is being far better controlled - due to greater research and investment, and better education leading to reduced transmission - than subspecies M: A in far poorer Africa.

So natural selection in the concrete case can show spatiotemporal organization. But actually natural selection and protein synthesis both show both simpler and more complex forms of organization. The HIV example is useful to show this. 
Dynamical relations are important to almost all mechanisms. These might be temporal organization, but they introduce a complexity that is concealed by failing to notice that they are vital. Understanding this undermines the apparent difference between protein synthesis and natural selection. Even spatiotemporal organization is dynamic organization not of a structure, but a functioning system. Dynamical organization is often left implicit, but it is ubiquitous. The relative speeds of things happening is vital even in simple cases. This makes HIV a successful retrovirus: "Viral RNA can use as much as $40 \%$ of total protein synthesis for the production of gag viral protein ... . HIV produces viral RNAs at a level that has the potential to inhibit or compete for host protein synthesis (Somasundaran \& Robinson 1988).' (Hutchinson 2001 p89.) A second example is that HIV can only produce AIDS by replicating faster than the immune system or drugs can kill it. The paper describes a complex dynamical play-off between HIV and the immune system, until HIV wins. If our immune system could produce new kinds of killer cells faster than HIV could kill them, the immune system would win. Modelling has also been used to understand the effects of selection: 'Mathematical models of the interaction between CD4+ cells and HIV-1 indicate that selection favors more virulent strains (Anderson 1989, 1991), and more virulent strains appear later in the asymptomatic or incubation period (Levy 1990, Fenyo et al 1989).' (Hutchinson 2001 p91.)

The HIV example also illustrates more complex forms of organization, since many are present. Spatiotemporal organization is relevant at all levels. The position and interaction of infected populations, organisms and cells matter. At a low level, organization of a cell, the way protein synthesis works, and the way a retrovirus works are important. But other forms of organization are relevant. The functioning of the immune system as the body's defence mechanism is important - to understand the seriousness of the problem when HIV disables it. Different strains of the virus act on the body in different ways: 'Although the pathogenesis of HIV is similar in those with HIV-2 and HIV-1 (De Cock et al 1990, Le Guenno et al 1991), the immunologic deficiency is less severe and the disease progression is slower in HIV-2 (Le Guenno et al 1991, Whittle et al 1994, Marlink et al 1994). The virulence of HIV-2 is known to vary significantly and range from relative attenuation to great pathogenicity. Differences in clinical manifestations may be partly related to genetic differences among infecting viral strains (Gao et al 1994).' (Hutchinson 2001 p96.) To achieve this, HIV's own highly specialised homeostatic mechanism is its attack on the immune system, to eventually disable it and leave the virus with a body in which it can do what it wants. Infection in a human population is highly susceptible to initial conditions of medical care and education which allow control over the spread of the virus.

These more complex forms of organization can be important even to protein synthesis. When examined in enough detail, the DNA damage response - a small part of the mechanisms which maintain the cell's DNA - is itself complex enough to invite simulation to understand it thoroughly. The interaction of genes even on this very small scale is sufficiently complex to create a system that can fall into different patterns of 
organization with sensitivity to initial conditions, and delicate feedback loops that can make it sensitive to perturbation. ${ }^{28}$

The apparent difference in predictability between natural selection and protein synthesis does not establish that one is a mechanism and one is not. It might be true that the more complex forms of organization are more often found in natural selection than protein synthesis, but this is a matter of degree - and one that has been exaggerated. Protein synthesis often operates in a closed system, in a cell, shielded from outside influences, while natural selection operates in an open system. These systems are more often highly sensitive to initial conditions, and to slight perturbations. ${ }^{29}$ This explains the differences we picked out in S1. It explains why natural selection more commonly produces unexpected results, yielding a need for replicate selection lines, and repeated experiment. Simulation can be extremely important to investigate such systems, so its usefulness to natural selection is obvious. But it is useful to protein synthesis too, which can include complex systems. This is the lesson of modelling the DNA damage response.

We have argued, against Skipper and Millstein, that natural selection in the concrete case does show spatiotemporal organization. We have said that Skipper and Millstein are right that no one form of organization is present in all cases of natural selection - but only organization understood at a certain level of abstraction. At a different level of abstraction, we can understand organization in general as when entities and activities each do something and do something together to produce the phenomenon. Understood this way, organization is important even to simple mechanisms, and complex forms of organization can be essential to the production of many phenomena, as we have illustrated with the HIV example. Both simpler and more complex forms of organization such as homeostatic mechanisms and feedback relations are present in both protein synthesis and natural selection. Organization gives us no reason to conclude that protein synthesis is a mechanism, and natural selection is not.

\section{Conclusion}

Mechanistic explanation begins with a specification of the phenomenon to be explained. At the least, this consists in an isolated description of a behaviour. Explanation then proceeds by identifying two kinds of parts - activities and entities - which contribute to producing that phenomenon. Entities and activities are both individuated in part by their roles in higher level mechanisms, so that they have role-functions derived from the characterisation of the phenomenon being explained. The explanation is not complete

\footnotetext{
${ }^{28}$ The DNA damage response is particularly worth modeling since it is involved both in healthy cells becoming cancerous, and in cancerous cells being difficult to kill. See the work of a group at the UCL Cancer Institute headed by Sylvia Nagl. This is best explored: http://www.ucl.ac.uk/cancer/researchgroups/cancer-systems-science/. (Accessed 3/3/10.) See Nagl (2006) for a thorough review of cancer bioinformatics.

${ }^{29}$ We find Barros' distinction between deterministic and stochastic mechanisms interesting. See Barros 2008 especially p309ff. But we think the distinction between natural selection and protein synthesis is not so clear-cut. Natural selection can sometimes be quite simple, and protein synthesis can sometimes be very complex.
} 
until the organization of those entities and activities - the relations they stand in which allow them to produce the phenomenon - is also identified. The process of discovery is of course not linear but iterative and messy. Along the way the phenomenon might be recharacterised, different entities and activities identified, and different forms of organization found.

Function has emerged as of vital importance in understanding mechanisms. Bechtel is exactly right when he writes: 'It bears emphasizing that the project of providing explanations, including mechanistic explanations, starts with the identification of a phenomenon. This is where the functioning structure gets determined, constraining what will count as a successful identification of relevant parts and operations and their organization.' (Bechtel 2006 p29.) With this deeper understanding, it can be seen that these three core elements of mechanistic explanation are present in both natural selection and protein synthesis. We hope we have also laid a groundwork for future work to expand these elements to other scientific fields.

We now have an explanation for the differences identified in S1 between the explanations offered and experimental work pursued in the two fields. Microstructure and the need for technology to allow its investigation is far more important in protein synthesis because such microstructure is commonly of vital functional relevance there. There is more need for mathematical modelling and simulation in natural selection because natural selection more often operates in systems requiring such modelling. But we have noted that these differences are merely of scale, applicable only in general, and not a deep division in kind. Structure -even microstructure - is also often of importance to natural selection; protein synthesis can require simulation.

We have argued against Skipper and Millstein. They claim that there are no parts of natural selection, only time-slices. We disagree, holding that natural selection indeed involves sub-mechanisms, which involve both entities and activities. We agree with Skipper and Millstein that organisms are sometimes relevant entities in natural selection, but hold that both organisms, and populations, are stable enough to be functionally relevant to natural selective explanations and so count as components of natural selection.

We agree with Skipper and Millstein that there is no one form of organization present in all cases of natural selection, but we do not think it follows that there is no mechanism of natural selection to be found. There are many forms of organization of parts that can be responsible for both protein synthesis and adaptation, and in different cases, different forms will be more important. Organization in general is any kind of relations among the parts by which they work together to produce the phenomenon in question, and at that level of abstraction, organization is present in both protein synthesis and natural selection. Just to reiterate, we have not addressed Skipper and Millstein's arguments concerning regularity - because we agree - or concerning productive continuity because that is a separate, and difficult, project.

It is important not to lose sight of what different forms of mechanistic organization - and indeed mechanistic explanation in general - have in common. Skipper and Millstein 
make an interesting comment on the methodology of philosophy of science: 'However, greater generality with respect to organization may not be desirable if it means sacrificing an understanding of the things that make mechanisms distinctive in particular fields, such as molecular biology.' (Skipper and Millstein 2005 p344.) They are quite right that an understanding of key differences in forms of organization should be sought, and not artificially smoothed out in a general account. Where certain forms of organization are more or less important to mechanisms in particular domains, that is interesting. But if a general understanding of organization is available, that should not be lost either. The overall conclusion of this paper is that the kinds of parts that should be sought, and the kinds of relations among them that explain phenomena, are empirical discoveries. Much detail about relevant parts and relations will be particular to a domain, or particular to a mechanism. This understanding should help in studying the structure of mechanistic explanation with respect to any domain.

\section{Acknowledgements}

We would like to thank the Leverhulme Trust for funding this work on mechanisms. We are also grateful to numerous colleagues and students at Kent, Bristol, and internationally for discussion of ideas used here. Particular thanks are due to Tudor Baetu, Federica Russo, Erik Weber, and an anonymous referee.

\section{References}

Adams, RL; Knowler, DP \& Leader, JT (1992). The Biochemistry of the Nucleic Acids (11 ${ }^{\text {th }}$ ed.). London: Chapman and Hall.

Barros, BD (2008). Natural Selection as a Mechanism. Philosophy of Science, 75, pp306-322.

Bechtel, W (2008). Mental Mechanisms: Philosophical perspectives on cognitive neuroscience. Oxford: Routledge.

Bechtel, W (2007). Reducing psychology while maintaining its autonomy via mechanistic explanations. In Maurice Schouten and Huib Looren de Jong (Eds.), The Matter of the Mind. Oxford: Blackwell.

Bechtel, W (2006). Discovering Cell Mechanisms: the Creation of Modern Cell Biology. Cambridge: CUP.

Bechtel, W \& Abrahamsen, A (2008). From Reduction Back to Higher Levels. In Love, BC; McRae, K \& Sloutsky, VM (Eds.). Proceedings of the 30th Annual Conference of the Cognitive Science Society (pp559-564). Austin, TX: Cognitive Science Society.

Bechtel, W \& Abrahamsen, A (2005). Explanation: a mechanist alternative. In Studies in History and Philosophy of Biological and Biomedical Sciences, 36, pp421-441.

Bell, G (2008). Selection: The Mechanism of Evolution ( $2^{\text {nd }}$ Edition). Oxford: OUP.

Bell, G (1997). Selection: The Mechanism of Evolution. NY, USA: Chapman and Hall.

Craver, C (2007). Explaining the Brain. Oxford: Clarendon Press.

Craver, C (2001). Role functions, mechanisms and hierarchy. In Philosophy of Science, 68(1), pp53-74.

Cummins, R (1975). Functional Analysis. In Journal of Philosophy, 72, pp741-765.

Darden, L (2006). Reasoning in Biological Discoveries. Cambridge: CUP.

Darden, L (2002). Strategies for Discovering Mechanisms: Schema Instantiation, Modular Subassembly, Forward/Backward Chaining. In Philosophy of Science, 69, ppS354-S365.

Darden, L \& Craver, C (2002). Strategies in the interfield discovery of the mechanism of protein synthesis. In Studies in History and Philosophy of Biological and Biomedical Sciences, 33, pp1-28.

Davies, PS (2000). Malfunctions. In Biology and Philosophy, 15, pp19-38.

Glennan, S (forthcoming). Productivity, relevance and natural selection. Forthcoming in Biology and Philosophy DOI 10.1007/s10539-008-9137-7 
Glennan, S (2002). Rethinking Mechanistic Explanation. In Philosophy of Science, 69, ppS342-S353.

Glennan, S (1996) Mechanisms and the Nature of Causation. In Erkenntnis, 44(1), pp49-71.

Godfrey-Smith, P (2009). Darwinian populations and Natural Selection. Oxford: OUP.

Hutchinson, JF (2001). The Biology and Evolution of HIV. In Annual Review of Anthropology, Vol. 30, pp85-108.

Illari, P \& Williamson, J (forthcoming). In Defense of Activities. Under review.

Illari, P \& Williamson, J (2010). Mechanisms are real and local. In Causality in the Sciences. Oxford, OUP.

Laubichler, M \& Wagner, G (2001). How Molecular is Molecular Developmental Biology? In Biology and Philosophy, 16, pp53-68.

Machamer, P (2004). Activities and Causation: The Metaphysics and Epistemology of Mechanisms. In International Studies in the Philosophy of Science, 18(1), pp27-39.

Machamer, P; Darden, L \& Craver, C (2000). Thinking about Mechanisms. In Philosophy of Science, 67, pp1-25.

Millikan, R (1984). Language, Thought, and Other Biological Categories. Cambridge: MIT Press.

Millikan, R (1989). In Defense of Proper Functions. In Philosophy of Science, 56, pp288-302.

Mitchell, SD (2003). Biological Complexity and Integrative Pluralism. Cambridge: CUP.

Nagl, S (2006). Cancer Bioinformatics: From Therapy Design to Treatment. London: Wiley's and Sons.

Neander, K (1991). Functions as Selected Effects: The Conceptual Analyst's Defense. In Philosophy of Science, 58, pp168-184.

Price, T (1998): Maternal and Paternal Effects in Birds: Effects on Offspring Fitness. In Mousseau, TJ \& Fox, CW (Eds.) Maternal Effects as Adaptations (pp202-226). Oxford: OUP.

Russo, F (2008). Causality and causal modelling in the social sciences: Measuring variations. Springer.

Skipper, RA (Jr.) \& Millstein, RL (2005). Thinking about evolutionary mechanisms: natural selection. In Studies in History and Philosophy of Biological and Biomedical Sciences, 36, pp327-347.

Voet, D and Voet, JG (2004). Biochemistry. USA: John Wiley and Sons.

Whitford, D (2005). Proteins: Structure and Function. Sussex: John Wiley and Sons Ltd.

Wright, L (1973). Functions. In Philosophical Review, 82, pp139-168. 